\title{
Relationship of homestead food production with night blindness among children below 5 years of age in Bangladesh
}

\author{
Ashley A Campbell ${ }^{1,2}$, Nasima Akhter ${ }^{3}$, Kai Sun ${ }^{1}$, Saskia de Pee ${ }^{2}$, Klaus Kraemer $^{4}$, \\ Regina Moench-Pfanner ${ }^{5}$, Jee $\mathrm{H} \mathrm{Rah}^{4}$, Jane Badham ${ }^{4,6}$, Martin W Bloem $^{2}$ and \\ Richard D Semba ${ }^{1, *}$ \\ 'Department of Ophthalmology, Johns Hopkins University School of Medicine, 400 North Broadway, M015, \\ Baltimore, MD 21287, USA: ${ }^{2}$ Nutrition Service, Policy, Strategy and Programme Support Division, World \\ Food Programme, Rome, Italy: ${ }^{3}$ Helen Keller International Asia Pacific, Dhaka, Bangladesh: ${ }^{4}$ SIGHT AND LIFE, \\ Basel, Switzerland: ${ }^{5}$ Global Alliance for Improved Nutrition, Geneva, Switzerland: ${ }^{6}$ JB Consultancy, Durban, \\ South Africa
}

Submitted 11 May 2010: Accepted 25 February 2011: First published online 4 May 2011

\begin{abstract}
Objective: To examine the relationship between homestead food production and night blindness among pre-school children in rural Bangladesh in the presence of a national vitamin A supplementation programme.

Design: A cross-sectional study.

Setting: A population-based sample of six rural divisions of Bangladesh assessed in the Bangladesh Nutrition Surveillance Project 2001-2005.

Subjects: A total of 158898 children aged 12-59 months.

Results: The prevalence rates of night blindness in children among those who did and did not receive vitamin A capsules in the last 6 months were $0.07 \%$ and $0 \cdot 13 \%$, respectively. Given the known effect of vitamin A supplementation on night blindness, the analysis was stratified by children's receipt of vitamin A capsules in the last 6 months. Among children who did not receive vitamin A capsules in the last 6 months, the lack of a home garden was associated with increased odds of night blindness (OR $=3 \cdot 16,95 \%$ CI 1.76, 5.68; $P=0 \cdot 0001$ ). Among children who received vitamin A capsules in the last 6 months, the lack of a home garden was not associated with night blindness (OR $=1 \cdot 28,95 \%$ CI $0 \cdot 71$, $2 \cdot 31 ; P=0 \cdot 4)$.

Conclusions: Homestead food production confers a protective effect against night blindness among pre-school children who missed vitamin A supplementation in rural Bangladesh.
\end{abstract}

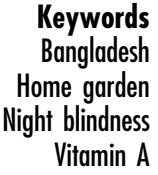

Keywords

Home garden

Vitamin A
Vitamin A deficiency remains a global public health problem. WHO recently reported that an estimated 190 million pre-school children are vitamin A deficient in low-income countries $^{(1)}$. Night blindness is one of the most common manifestations of vitamin A deficiency, which is associated with a number of adverse health conditions such as decreased immunity and increased mortality, especially from measles, diarrhoea and malaria ${ }^{(2)}$. Many developing countries utilize complementary and overlapping strategies to reduce vitamin A deficiency among children, as well as among other members of the population, including periodic high-dose vitamin A supplementation, fortification of foods with vitamin A and promotion of homestead food production.

In Bangladesh, it has been shown previously that locally produced fruit and vegetables rich in provitamin $\mathrm{A}$ provide a valuable source of maternal vitamin A intake, particularly in communities in which vitamin A-rich food is scarce ${ }^{(3,4)}$. In 2005, 65\% of households in rural Bangladesh had a traditional home garden, and only $21 \%$ had no garden ${ }^{(5)}$. In addition, it has been shown in South Africa that production of yellow and dark-green leafy vegetables through the initiation of home-gardening programmes significantly improved the vitamin A status of pre-school children ${ }^{(6,7)}$. In a national vitamin A survey of 24000 households in Bangladesh in 1997, it was shown that possession of a home garden was associated with a more than twofold reduced odds of having night blindness, especially in mothers ${ }^{(8)}$. The advantage of having gardens is that even very poor and landless people can produce food, whether it be on small patches of rented homestead land, roof tops, in containers or on roadsides, and with limited economic resources ${ }^{(8,9)}$. Home gardens may represent a more sustainable solution compared with periodic high-dose vitamin A supplementation. 
Although the role of universal distribution of vitamin A capsules in combating vitamin A deficiency in Bangladesh is well known ${ }^{(10)}$, the importance of home garden ownership has been less well characterized. We hypothesized that, among children who did not receive vitamin A recently, the risk of night blindness is greater in households that do not have a home garden compared with those having a home garden. To address this hypothesis we characterized the relationship between household possession of a home garden and night blindness in children in rural Bangladesh in the context of receiving or not receiving vitamin A capsules.

\section{Participants and methods}

Data were collected from households that were selected by the Nutrition Surveillance Project (NSP) in Bangladesh from February 2001 through December 2005. The NSP was based on the conceptual framework of UNICEF on the causes of malnutrition ${ }^{(11)}$, with the underlying principle of providing information to guide policies and programmes to improve nutrition in Bangladesh and other developing countries ${ }^{(12)}$. The NSP was established by Helen Keller International and by the Institute of Public Health Nutrition (IPHN) of the Government of Bangladesh in $1989^{(13)}$. The NSP was based on stratified multistage cluster sampling of households in sub-districts of administrative divisions of the country in rural areas and slum areas of large cities. For the purpose of the present analysis, only data from rural areas were considered. Rural data were collected from four sub-districts of each of the six divisions of Bangladesh - Barisal, Chittagong, Dhaka, Khulna, Rajshahi and Sylhet. Although Chittagong Hill Tracts is administratively under the Chittagong Division, the Hill Tracts were treated as a special sampling frame to provide data specifically for that area of the country for the purposes of the present analysis. Data were collected every 2 months by twoperson field teams employed by the IPHN and various non-governmental organizations. Throughout the period of data collection, the sub-districts remained the same but new households were selected for every round of data collection. For each of the six divisions in each round, four sub-districts were randomly chosen. From each of the sub-districts fifteen mouza (administrative districts) were randomly selected, and from each mouza twenty-five households were systematically sampled. In total, during each round, data were collected from about 10500 households. A structured coded questionnaire was used to record data on children aged 0-59 months, including anthropometric measurements, date of birth and sex. The mother of the child or any other adult member of the household was asked to provide information on the household's composition, maternal age, maternal education and weekly household expenditures, along with other socio-economic, environmental, sanitation and health indicators.

In the present study, families who had at least one child aged 12-59 months were included. Families were excluded if there was only one child $<12$ months of age, because night blindness is more difficult to assess in infants. Birth dates of the children were obtained from birth certificates or from other records. When not available, the birth dates were estimated using a calendar of local and national events and converted to the Gregorian calendar. In order to assess night blindness, data collectors used local terms such as 'ratkana', 'naitan', 'lali andha', 'aloandhari', etc. They were also asked to mention symptoms of this problem to verify whether night blindness was a familiar term in the area. This was used as a clinical indicator of vitamin A deficiency. Children who also had trouble seeing during the daytime were excluded. A history of night blindness has been shown to be a reliable indicator of vitamin A deficiency, given that a high proportion of children reporting night blindness have low serum retinol levels also ${ }^{(14)}$. In households with more than one child having night blindness, only the youngest was included (i.e. households were not counted more than once). Families that were defined as having a home garden in the last 12 months were those who reported having a traditional garden, improved garden or a developed garden in the last 12 months. Traditional gardens typically grow seasonal gourd-type vegetables in scattered plots, whereas improved gardens are those that grow seasonal vegetables in fixed plots. Developed gardens grow a wide variety of fruit and vegetables (usually more than six varieties) throughout the year.

The participation rate of families in the surveillance system was $>97 \%$ and the main reason for non-response was that the family had moved out of the area or was absent at the time of interviews. Non-response because of refusal to participate in the surveillance system was very low $(<1 \%)$.

The study protocol complied with the principles enunciated in the Helsinki Declaration ${ }^{(15)}$. The field teams were instructed to explain the purpose of the NSP and data collection to each child's mother or caregiver, and, if present, to the father and/or household head; data collection proceeded only after written informed consent was obtained. Participation was voluntary and all participants were free to withdraw at any stage of the interview. The protocol was approved by the Ethical Review Committee of the Bangladesh Medical Research Council, and the plan for secondary data analysis was approved by the Institutional Review Board of the Johns Hopkins School of Medicine.

Categorical variables were compared using the $\chi^{2}$ test. Separate multivariable models were used to examine the relationship between the family having a home garden in the last 12 months and night blindness in children, 
stratified by the child's receipt of vitamin A supplementation in the last 6 months. Variables that were significantly associated with night blindness in children were included in the respective, final multivariable models. Population-based weighting was used in all analyses to account for differences in population size among the various divisions. The level of significance in the present study was $P<0 \cdot 05$.

\section{Results}

Of 158898 children aged 12-59 months in rural Bangladesh, 45606 (29\%) did not receive vitamin A capsules in the last 6 months. Among children who did and did not receive vitamin A capsules in the last 6 months, the proportions of those with night blindness were $0.07 \%$ and $0 \cdot 13 \%$, respectively. The demographic characteristics of households with and without night blindness in children are shown in Table 1 . Households with mothers who were less educated, had younger children, had a lower weekly per capita household expenditure and did not have a home garden were more likely to have a child with night blindness. The prevalence of night blindness was highest in Barisal and Rajshahi and lowest in Dhaka and Chittagong divisions.

Multivariable models are shown for the relationship between having a home garden and night blindness in children, stratified by receipt of vitamin A supplementation in the last 6 months (Table 2). Among children who did not receive vitamin $\mathrm{A}$, the lack of ownership of a home garden was related to night blindness in a

Table 1 Risk factors for night blindness in children aged 12-59 months from families in rural Bangladesh

\begin{tabular}{|c|c|c|c|c|c|c|}
\hline \multirow[b]{2}{*}{ Characteristic } & \multicolumn{3}{|c|}{ Non-vitamin A recipients } & \multicolumn{3}{|c|}{ Vitamin A recipients } \\
\hline & Cases/total & $\%$ & $P$ & Cases/total & $\%$ & $P$ \\
\hline \multicolumn{7}{|l|}{ Maternal age (years) } \\
\hline$\leq 22$ & $11 / 11199$ & $0 \cdot 1$ & \multirow[t]{4}{*}{$0 \cdot 34$} & $14 / 25864$ & $0 \cdot 1$ & \multirow[t]{4}{*}{0.6} \\
\hline $23-26$ & $14 / 12772$ & $0 \cdot 1$ & & $20 / 32578$ & $0 \cdot 1$ & \\
\hline $27-30$ & $16 / 9514$ & $0 \cdot 2$ & & $21 / 25126$ & $0 \cdot 1$ & \\
\hline$\geq 31$ & $20 / 12112$ & $0 \cdot 2$ & & $19 / 29677$ & $0 \cdot 1$ & \\
\hline \multicolumn{7}{|c|}{ Maternal education (years) } \\
\hline 0 & $42 / 23111$ & $0 \cdot 2$ & \multirow[t]{4}{*}{0.006} & 46/49 971 & $0 \cdot 1$ & \multirow[t]{4}{*}{0.006} \\
\hline $1-6$ & $16 / 13586$ & $0 \cdot 1$ & & $22 / 36759$ & $0 \cdot 1$ & \\
\hline $7-9$ & $2 / 6478$ & 0.03 & & $4 / 19065$ & 0.02 & \\
\hline$\geq 10$ & $0 / 2100$ & $0 \cdot 0$ & & $2 / 6806$ & 0.03 & \\
\hline \multicolumn{7}{|c|}{ Ownership of a home garden in the last 12 months } \\
\hline No & $20 / 9112$ & 0.2 & \multirow[t]{2}{*}{0.01} & $12 / 20940$ & $0 \cdot 1$ & \multirow[t]{2}{*}{0.62} \\
\hline Yes & $41 / 36494$ & $0 \cdot 1$ & & $62 / 92406$ & $0 \cdot 1$ & \\
\hline \multicolumn{7}{|l|}{ Garden type } \\
\hline None & $30 / 8939$ & $0 \cdot 34$ & \multirow[t]{4}{*}{$<0.0001$} & $20 / 21118$ & 0.09 & \multirow[t]{4}{*}{$0 \cdot 85$} \\
\hline Traditional & $31 / 28929$ & $0 \cdot 11$ & & $64 / 73476$ & 0.09 & \\
\hline Improved & $10 / 5394$ & $0 \cdot 19$ & & $15 / 15520$ & $0 \cdot 10$ & \\
\hline Developed & $3 / 1245$ & $0 \cdot 24$ & & 2/3818 & 0.05 & \\
\hline \multicolumn{7}{|l|}{ Child's sex } \\
\hline Female & $31 / 21922$ & $0 \cdot 1$ & \multirow[t]{2}{*}{0.67} & $35 / 54115$ & $0 \cdot 1$ & \multirow[t]{2}{*}{0.94} \\
\hline Male & $30 / 23684$ & $0 \cdot 1$ & & $39 / 59177$ & $0 \cdot 1$ & \\
\hline \multicolumn{7}{|l|}{ Child's age (months) } \\
\hline $12-23$ & $11 / 14542$ & $0 \cdot 1$ & \multirow[t]{4}{*}{0.07} & $10 / 28215$ & 0.04 & \multirow[t]{4}{*}{0.04} \\
\hline 24-35 & $18 / 11410$ & $0 \cdot 2$ & & $20 / 30859$ & $0 \cdot 1$ & \\
\hline $36-47$ & $19 / 9679$ & $0 \cdot 2$ & & $24 / 27369$ & $0 \cdot 1$ & \\
\hline $48-59$ & 9/6969 & $0 \cdot 1$ & & $19 / 19194$ & $0 \cdot 1$ & \\
\hline \multicolumn{7}{|c|}{ Number of individuals eating food prepared from the same kitchen } \\
\hline $0-4$ & $20 / 16080$ & $0 \cdot 1$ & \multirow[t]{2}{*}{0.69} & $28 / 40107$ & $0 \cdot 1$ & 0.66 \\
\hline$>4$ & $41 / 29524$ & $0 \cdot 1$ & & $46 / 73185$ & $0 \cdot 1$ & \\
\hline Weekly per capita house & & & & & & \\
\hline 1 & $9 / 8421$ & $0 \cdot 1$ & 0.65 & $19 / 19003$ & $0 \cdot 1$ & $0 \cdot 15$ \\
\hline 2 & $11 / 8941$ & $0 \cdot 1$ & & $14 / 21484$ & $0 \cdot 1$ & \\
\hline 3 & $16 / 9282$ & $0 \cdot 2$ & & $18 / 23204$ & $0 \cdot 1$ & \\
\hline 4 & $15 / 9553$ & $0 \cdot 2$ & & $12 / 24362$ & 0.05 & \\
\hline 5 & $10 / 9408$ & $0 \cdot 1$ & & $11 / 25239$ & 0.04 & \\
\hline Division of country & & & & & & \\
\hline Barisal & $6 / 5987$ & $0 \cdot 1$ & $<0.0001$ & $11 / 16776$ & $0 \cdot 1$ & 0.007 \\
\hline Chittagong & $3 / 6535$ & $0 \cdot 1$ & & $5 / 16489$ & 0.03 & \\
\hline Chittagong Hill Tracts & $11 / 6280$ & $0 \cdot 2$ & & $8 / 16413$ & 0.05 & \\
\hline Dhaka & 0/5409 & $0 \cdot 0$ & & $6 / 16945$ & 0.04 & \\
\hline Khulna & $9 / 5760$ & $0 \cdot 2$ & & $11 / 16694$ & $0 \cdot 1$ & \\
\hline Rajshahi & $20 / 6330$ & $0 \cdot 3$ & & $22 / 16823$ & $0 \cdot 1$ & \\
\hline Sylhet & $12 / 9305$ & $0 \cdot 1$ & & 11/13152 & $0 \cdot 1$ & \\
\hline
\end{tabular}

${ }^{*}$ Quintile 1 (the reference quintile) is the lowest and quintile 5 is the highest. 
Table 2 Separate multivariable models for the relationship of ownership of a home garden in the last 12 months with night blindness in children with and without vitamin A coverage in the last 6 months*

\begin{tabular}{lccl}
\hline Vitamin A coverage & OR & $95 \% \mathrm{Cl}$ & \multicolumn{1}{c}{$P$} \\
\hline Child received vitamin A & 1.15 & $0.64,2.07$ & 0.6 \\
Child did not receive vitamin A & 2.77 & $1.55,4.97$ & 0.0006 \\
\hline
\end{tabular}

*Separate multivariable models are each adjusted for maternal education, child's age, weekly per capita household expenditure, location, height-for-age $Z$-score, season, diarrhoeal morbidity and breast-feeding status.

multivariable logistic regression model adjusting for maternal education, child's age, weekly per capita household expenditure, location, season of data collection, heightfor-age $Z$-score, diarrhoeal morbidity and breast-feeding status. The relationship between having a home garden and night blindness in children was not significant among children who received vitamin A supplementation in the last 6 months. Additional multivariable models were performed to examine the relationship between garden type and night blindness in children, stratified by receipt of vitamin A supplementation in the last 6 months, adjusting for potential confounders. Among children who did not receive vitamin A, the risk of having night blindness with a traditional, improved and developed garden compared with no garden was: OR $=0 \cdot 30(95 \%$ CI $0 \cdot 16,0 \cdot 60 ; P=0 \cdot 0002) ; \mathrm{OR}=0.43(95 \% \mathrm{CI} 0 \cdot 19,0 \cdot 99$; $P=0.05)$; and $\mathrm{OR}=0.35(95 \%$ CI $0 \cdot 11,1 \cdot 16 ; P=0.09)$, respectively. Among children who received vitamin $\mathrm{A}$, the risk of having night blindness with a traditional, improved and developed garden compared with no garden was: $\mathrm{OR}=0.72(95 \%$ CI $0 \cdot 39,1 \cdot 32 ; P=0 \cdot 29) ; \mathrm{OR}=0 \cdot 97(95 \%$ CI $0 \cdot 42,2 \cdot 25 ; P=0 \cdot 94)$; and $\mathrm{OR}=1 \cdot 01$ (95\% CI $0 \cdot 23$, $4 \cdot 54 ; P=0 \cdot 98)$, respectively.

\section{Discussion}

The present study shows that the odds of night blindness among pre-school children who had not received vitamin A capsules were higher when the household did not have a home garden. Conversely, no significant relationship was found between having a home garden and night blindness among children who received high-dose vitamin A capsules in the last 6 months.

These findings suggest that home gardens provide added protection against clinical vitamin A deficiency among children who miss vitamin A supplementation through the national vitamin A supplementation programme. The health benefits of homestead food production include those from direct consumption of foods that are good sources of vitamin A, either produced in the garden directly or acquired with income generated from selling some of the garden's produce, or through reduced morbidity created indirectly by the garden's produce, such as better health care, education, housing or clothes ${ }^{(8)}$.
In addition, since homestead food production is traditionally a woman's task, it can lead to empowerment of women, which can result in an increase in the household's expenditure on food and health care ${ }^{(16)}$. Home gardens had no apparent protective effect against night blindness in children who received vitamin A capsules, which suggests that the capsules already provided sufficient vitamin A to prevent night blindness. Because the vitamin A capsule supplementation programme does not have $100 \%$ coverage and relies on government's goodwill and financial support, the combination of home gardens and vitamin A capsule coverage is important for children and may be more sustainable ${ }^{(17)}$. As this concept has been shown only in urban settings, there is a need for further substantiation in rural environments. The home garden also provides vitamin A to other household members. To provide adequate amounts of vitamin A, however, animal sources of vitamin $\mathrm{A}$ and fortified foods are also required because of increased bioavailability and bioconversion ${ }^{(8)}$.

Traditional gardens appear to confer the best protection from night blindness to children when compared with other types of gardens and no garden at all. Although one would expect the improved garden to be associated with the best protection, it is possible that there was limited power to consider this relationship since it had the smallest sample size.

The prevalence of night blindness was lower among children who received vitamin A capsules in the last 6 months. This observation is consistent with previous studies that showed that vitamin A supplementation of $60 \mathrm{mg}$ every 4-6 months reduces the appearance of new cases of xerophthalmia among pre-school children by $60-90 \%{ }^{(18-21)}$. Moreover, the prevalence of night blindness among children who did not receive vitamin A supplementation in the last 6 months was $0 \cdot 13 \%$, which is lower than previous estimates. For example, in 1982-1983 the prevalence of night blindness in children was $3.6 \%$ in rural settings and $2 \cdot 8 \%$ in urban settings ${ }^{(22)}$. This could be due to a number of factors. Over the past 20 years, there has been increasing coverage of the vitamin A capsule distribution project and improvements in overall socio-economic status. Thus, even those children who had not received vitamin $\mathrm{A}$ in the last 6 months could have received vitamin $\mathrm{A}$ in previous rounds. This would lead to an underestimation in the prevalence of the condition and could mean that the present risk estimates for the benefit of a home garden are potentially larger than reported, making the current estimates of risk conservative.

The analyses adjusted for factors that have previously been associated with night blindness in children (i.e. lower maternal education, younger children and location) and also for differences in socio-economic status using weekly per capita household expenditure. The association between the family having a home garden in the last 12 months and night blindness in children was strong 
among children without vitamin A supplementation even after adjusting for these factors. The strengths of the present study include the large sample size and a relatively extensive database pertaining to demographic and socio-economic indicators.

The present study has some limitations. The data from the NSP are cross-sectional. There could be unmeasured changes over time between ownership of a home garden at the time the data were collected and the conditions that originally led to the development of night blindness in children. In an attempt to overcome this limitation, however, ownership of a home garden in the last 12 months was chosen as the independent variable rather than current ownership of a garden, even though both were available in the NSP questionnaire. Further, the gap between production and consumption of the products grown from home gardens is unknown. This information will be valuable in future studies in order to design policy more specifically. Finally, although Bangladesh is fairly representative of a rural economy in South Asia, findings from the present study cannot necessarily be extrapolated to other countries. Future studies are needed to corroborate these findings in other settings in developing countries.

In conclusion, the presence of a home garden reduced the risk of night blindness among pre-school children, but only among those who did not receive vitamin A supplementation.

\section{Acknowledgements}

The present paper was supported by a writing workshop at Kappel-am-Albis, Switzerland, funded by Sight and Life, the humanitarian initiative of DSM. R.D.S. was funded in part by a Lew Wasserman Merit Award from Research to Prevent Blindness. The authors have no conflict of interest to declare. R.D.S., S.D.P. and M.W.B. conceived the topic; K.S. analysed the data; M.W.B. conducted data collection in Bangladesh; and K.K. and R.D.S. were involved in sourcing of funds for the study. All authors contributed to the writing of the manuscript.

\section{References}

1. World Health Organization (2009) Global Prevalence of Vitamin A Deficiency in Populations at Risk 1995-2005: WHO Global Database on Vitamin A Deficiency. Geneva: WHO.

2. West KP Jr \& Darnton-Hill I (2008) Vitamin A deficiency. In Nutrition and Health in Developing Countries, 2nd ed., pp. 377-434 [RD Semba and MW Bloem, editors]. Totowa, NJ: Humana Press.

3. Bloem MW, Huq N, Gorstein J et al. (1996) Production of fruits and vegetables at the homestead is an important source of vitamin A among women in rural Bangladesh. Eur J Clin Nutr 50, Suppl. 3, S62-S67.

4. Talukder A, Kiess L, Huq $\mathrm{N}$ et al. (2000) Increasing the production and consumption of vitamin A-rich fruits and vegetables: lessons learned in taking the Bangladesh homestead gardening programme to a national scale. Food Nutr Bull 21, 165-172.

5. Helen Keller International \& Institute of Public Health Nutrition (2006) Bangladesh in Facts and Figures: 2005 Annual Report of the Nutritional Surveillance Project. Dhaka, Bangladesh: HKI.

6. Faber M, Venter SL \& Benade AJ (2002) Increased vitamin A intake in children aged $2-5$ years through targeted homegardens in a rural South African community. Public Health Nutr 5, 11-16.

7. Faber M, Phungula MA, Venter SL et al. (2002) Home gardens focusing on the production of yellow and darkgreen leafy vegetables increase the serum retinol concentrations of 2-5-yr-old children in South Africa. Am J Clin Nutr 76, 1048-1054.

8. de Pee S, Talukder A \& Bloem MW (2008) Homestead food production for improving nutritional status and health. In Nutrition and Health in Developing Countries, 2nd ed., pp. 753-780 [RD Semba and MW Bloem, editors]. Totowa, NJ: Humana Press.

9. Miura S, Kunii O \& Wakai S (2003) Home gardening in urban poor communities of the Philippines. Int J Food Sci Nutr 54, 77-88

10. Bloem MW, Hye A, Wijnroks M et al. (1995) The role of universal distribution of vitamin A capsules in combating vitamin A deficiency in Bangladesh. Am J Epidemiol 142, 843-855.

11. de Pee S \& Bloem MW (2001) Assessing and communicating impact of nutrition and health programs. In Nutrition and Health in Developing Countries, pp. 483-506 [RD Semba and MW Bloem, editors]. Totowa, NJ: Humana Press.

12. Mason JB, Habicht JP, Tabatabai H et al. (1984) Nutritional Surveillance. Geneva: WHO.

13. Bloem MW, Moench-Pfanner R \& Panagides D (editors) (2003) Health and Nutritional Surveillance for Development. Singapore: Helen Keller Worldwide.

14. Sommer A, Hussaini G, Muhilal et al. (1980) History of night blindness: a simple tool for xerophthalmia screening. Am J Clin Nutr 22, 887-891.

15. World Medical Association (2001) World Medical Association Declaration of Helsinki: ethical principles for medical research involving human subjects. Bull World Health Organ 79, 373-374.

16. Bushamuka VN, de Pee S, Talukder A et al. (2005) Impact of a homestead gardening program on household food security and empowerment of women in Bangladesh. Food Nutr Bull 26, 17-25.

17. Moskow A (1999) The contributions of urban agriculture to gardeners, their households and surrounding communities: The case of Havana, Cuba. In For Hunger-Proof Cities: Sustainable Urban Food Systems, pp. 77-84 [M Koc, R MacRae, L Mougeot et al., editors]. Ottawa: CRDI.

18. Sinha DP \& Bang FB (1976) The effect of massive doses of vitamin A on the signs of vitamin A deficiency in preschool children. Am J Clin Nutr 29, 110-115.

19. Djunaedi E, Sommer A, Pandji A et al. (1988) Impact of vitamin A supplementation on xerophthalmia. A randomized controlled community trial. Arch Ophthalmol 106, $218-222$

20. Sommer A (1995) Nutritional Blindness: Xerophthalmia and Keratomalacia. New York: Oxford University Press.

21. Katz J, West KP Jr, Khatry SK et al. (1995) Impact of vitamin A supplementation on prevalence and incidence of xerophthalmia in Nepal. Invest Ophthalmol Vis Sci 36, 2577-2583.

22. Cohen N, Rahman H, Sprague J et al. (1985) Prevalence and determinants of nutritional blindness in Bangladeshi children. World Health Stat Q 38, 317-330. 